\title{
Gradhiva
}

GRADHIV

Revue d'anthropologie et d'histoire des arts

11 | 2010

Grands hommes vus d'en bas

\section{Les vérités contrariées du totem Lincoln}

The Vexed Truths of the Lincoln Totem

\section{Nicolas Menut}

\section{OpenEdition}

Journals

Édition électronique

URL : http://journals.openedition.org/gradhiva/1674

DOI : $10.4000 /$ gradhiva. 1674

ISSN : 1760-849X

\section{Éditeur}

Musée du quai Branly Jacques Chirac

\section{Édition imprimée}

Date de publication : 19 mai 2010

Pagination : 100-117

ISBN : 978-2-35744-025-8

ISSN : 0764-8928

Référence électronique

Nicolas Menut, «Les vérités contrariées du totem Lincoln », Gradhiva [En ligne], 11 | 2010, mis en ligne le 19 mai 2013, consulté le 01 mai 2019. URL : http://journals.openedition.org/gradhiva/1674 ; DOI : 10.4000/gradhiva.1674 


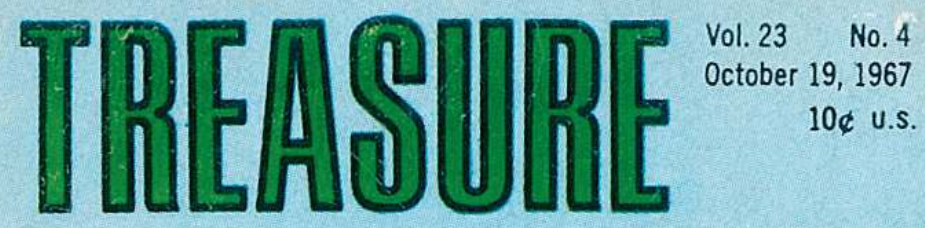

DF FUN AND FACT

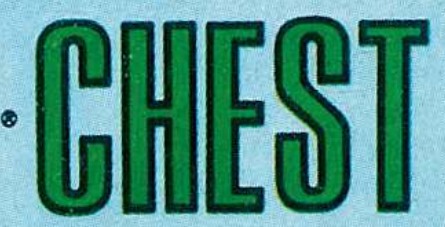




\section{Les vérités contrariées du totem Lincoln*}

Nicolas Menut

Fig. 1 Lincoln's Totem Pole, Treasure Chest, vol 23, $\mathrm{n}^{\circ} 4$ 19 octobre 1967 (couverture). Avec l'aimable autorisation de la succession Lloyd Ostendorf, Dayton, $\mathrm{OH}, \mathrm{USA}$.

\footnotetext{
- $\bullet$
}

* Mes remerciements à Julien Bonhomme pour sa précieuse relecture.

1. À la suite des travaux de l'anthropologue allemand, plusieurs publications isolées tentèrent cependant, avec plus ou moins de réussite, de lui faire écho. Cf. Julia Blackburn, The White Men. The First Response of Aboriginal Peoples to the White Man. New York, Times Books, 1979 et Exotic Europeans. Londres, The South Bank Center, 1991 ; Anna Jackson, Encounters. The Meeting of Asia and Europe. Londres, Victoria and Albert Museum Publications, 2004.
Depuis la publication en 1937 de l'ouvrage fondateur de Julius Lips, The savage hits back, force est de reconnaître que les anthropologues et les historiens de l'art ne se sont guère intéressés à la représentation de l'homme blanc dans les arts non occidentaux, privilégiant, dans un renversement ethnocentriste, l'étude du regard européen sur les populations et les productions artistiques non occidentales.1. Pour autant, les analyses développées par Lips, pour novatrices qu'elles soient, n'en demeurent pas moins sujettes à caution. En effet, la nature même des objets étudiés par l'anthropologue allemand, leurs conditions de production et leurs fonctions au sein des sociétés qui les ont réalisés, sans oublier le propre regard de l'ethnologue ou de l'historien, ont tendance à favoriser une multiplicité de grilles de lecture où il n'est pas rare que les versions, en fonction du point de vue où l'on se place, divergent, voire s'opposent.

Le cas d'un totem tlingit, communément baptisé "totem Lincoln", illustre parfaitement ces divergences d'interprétation : en l'espace d'un siècle, plusieurs commentaires divergents au sujet dudit mât ont été produits, éclairant d'un jour inédit et ambigu l'histoire de cette population indigène établie dans le sud-est de l'Alaska. Un totem Lincoln, érigé en 1940, est aujourd'hui visible au Saxman Totem Park à Ketchikan, en Alaska. Il s'agit en réalité d'une copie de l'œuvre originale réalisée par des sculpteurs amérindiens recrutés par le Civilian Conservation Corps au cours de la Grande Dépression des années 1930 afin de préserver et de restaurer une partie du patrimoine des peuples indigènes d'Alaska. Du fait de son délabrement, les autorités décidèrent de déplacer le totem original qui se trouvait dans le village de Tongass depuis les années 1880 et de désolidariser la figure de Lincoln du reste du mât à des fins de conservation. Bien que l'on ne dispose pas d'informations précises à ce sujet, les photographies d'époque permettent de constater qu'un corbeau aux ailes déployées avait été sculpté à la base du 


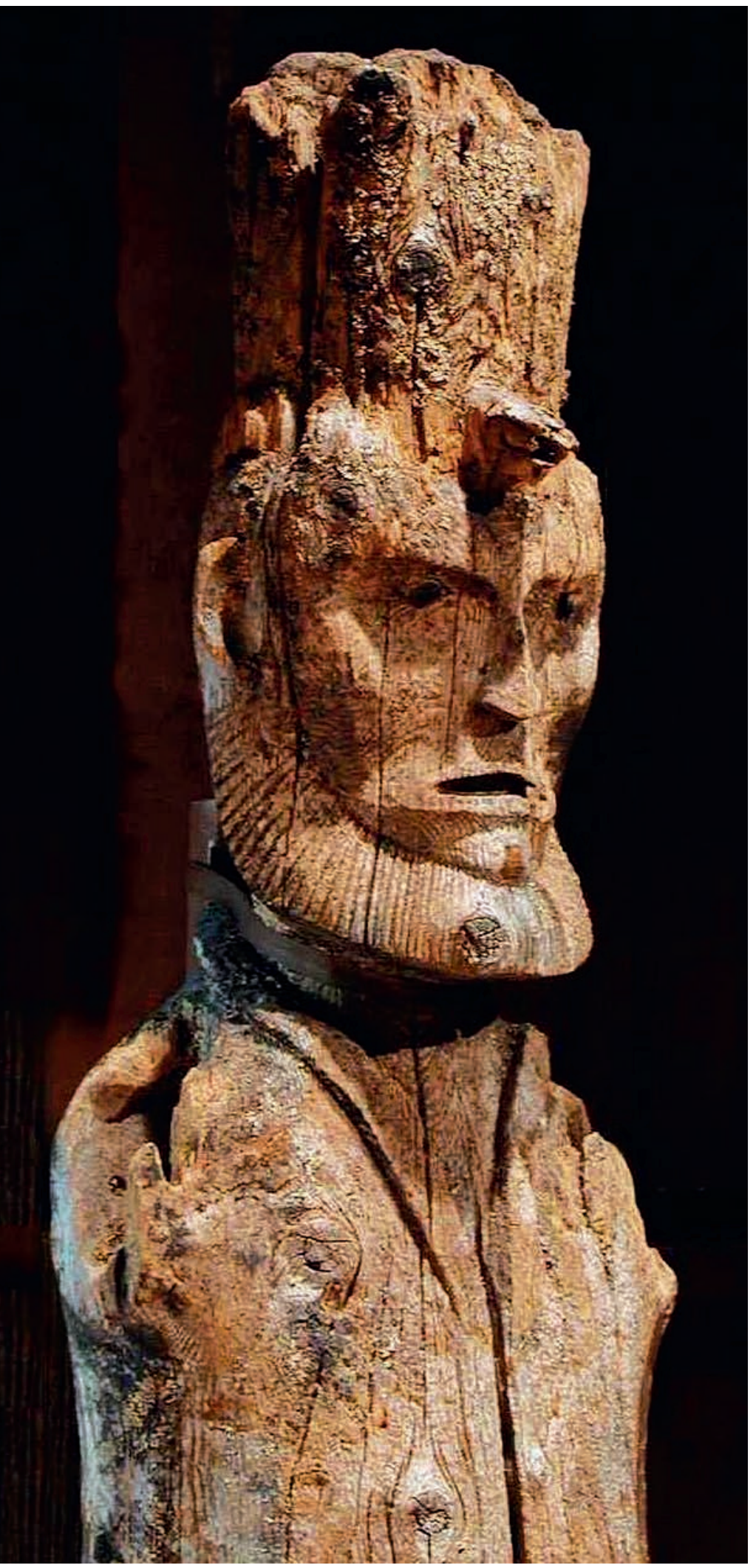

Fig. 2 Totem en bois représentant Abraham Lincoln-Tlingit, Tongass Island @ Alaska State Museum, Juneau [II-B-833]. totem. Le mât était, par ailleurs, vierge de toute autre illustration et seule, à son sommet, trônait la figure du président Lincoln. Il ne subsiste aujourd'hui de l'œuvre originale qu'une sculpture érodée en bois de cèdre rouge représentant Abraham Lincoln en pied (d'une hauteur de $183 \mathrm{~cm}$ et d'une largeur de $45 \mathrm{~cm}$ ), appartenant aux collections de l'Alaska State Museum à Juneau.

La figure d'Abraham Lincoln, seizième président des États-Unis d'Amérique, est aujourd'hui intimement liée à deux événements qui ont profondément marqué la société américaine : la résolution de la guerre de Sécession et l'abolition de l'esclavage. Lincoln fut assassiné le 15 avril 1865 et la Nation reconnaissante n'a cessé, dès lors, de lui rendre hommage : des pièces de monnaie et des billets furent émis à son effigie, la capitale du Nebraska adopta son nom tandis que, de 1927 à 1941, le sculpteur Gutzon Borglum, aidé par quelque quatre cents ouvriers, gravait son portrait dans la roche du mont Rushmore. Ces hommages post mortem inscrivent ainsi la figure de Lincoln dans une histoire nationale dont il apparaît comme l'une des icônes les plus importantes. Peut-on pour autant affirmer que sa présence au sommet d'un totem tlingit relève de la même démarche? Entre 1924 et 1997, plusieurs articles sont publiés qui, loin de s'entendre autour d'une version commune, avancent des interprétations contradictoires, voire incompatibles. Trois théories s'affrontent comme autant de voix discordantes faisant référence à l'histoire coloniale des États-Unis et à sa perception par les Indiens tlingit. Nous verrons que ces divergences d'interprétation sont fondamentalement politiques car elles portent sur la légitimation du pouvoir blanc et sa critique indigène. Ce n'est pas seulement la perception indigène du grand homme blanc qui est en jeu dans cette affaire; c'est, à un niveau de complexité supérieure, à la manière d'une mise en abyme, les regards divergents portés par des Blancs et des Indiens sur cette perception autochtone du Blanc. Si les premières lectures du totem Lincoln sont le fait de Blancs qui légitiment leur propre pouvoir sur les Indiens en s'arrogeant le monopole de l'interprétation du regard indigène sur eux, ces interprétations sont bientôt mises à mal par des Indiens qui cherchent ainsi à se réapproprier le sens de leur propre regard. 


\section{Quand l'homme blanc s'invite sur les totems}

La présence d'une figure du pouvoir fédéral américain - qu'il s'agisse d'un président, d'un gouverneur, voire d'un haut gradé militaire - sur un totem n'est pas un cas exceptionnel. Après le rachat de l'Alaska à la Russie par le gouvernement des États-Unis en 1867, plusieurs totems exhibant des figures de personnalités non autochtones furent ainsi recensés au gré des enquêtes de l'administration américaine. L'anthropologue canadien Marius Barbeau, dans son ouvrage Totem Poles (1964), consacreplusieurs pagesàcesujet.OutreletotemLincoln,ilévoqueplusieurs mâts présentant des figures d'origine européenne, parmi lesquels le Chief Skoolka's Gratitude Pole et le Chief Skowl's Totem Pole. Le premier totem était déjà mentionné dans un article de l'anthropologue américain Georges T. Emmons en 1914 :

«Dans le village haïda de Howkan, sur l'île du prince de Galles, se trouvait encore en 1914 le totem du chef Skoolka au sommet duquel figurait un personnage en uniforme avec une longue barbe. Il s'agissait d'un officier militaire de Sitka qui avait prodigué quelques bienfaits à la famille dudit chef. » (1914: 66-67)

Dans un court article, Robin $\mathrm{K}$. Wright, responsable du département d'art amérindien au Burke Museum, multiplie les spéculations quant aux raisons de la présence de cette figure d'Occidental sur ce totem, mais se garde, faute d'éléments tangibles, de toute affirmation péremptoire ${ }^{2}$. Elle évoque notamment le témoignage de l'écrivain et géographe Eliza Ruhamah Scidmore, qui interrogea Skoolka au début des années 1880. Ce dernier lui avait expliqué que les images figurant sur le totem racontaient l'histoire de l'une de ses ancêtres :

" [C']était une femme importante appartenant au clan de l'aigle. Elle partit chercher des œufs de saumon, et quand elle remonta son canoë sur la plage, à son retour, elle en avait rempli plusieurs paniers. Ne voyant pas ses deux petits enfants, elle les appela, mais ils coururent se cacher. Plus tard, elle les appela à nouveau, et ils lui répondirent de la forêt avec des voix de corneille. Ses pires craintes virent le jour lorsqu'elle réalisa qu'un homme blanc, un Bostonien [Boston man], les avait enlevés et conduits à bord d'un bateau. Les deux orphelins ne revinrent jamais auprès de leur peuple. Cette histoire d'enlèvement fut transmise de génération en génération dans la famille de Skulka, et ce visage barbu sur le totem est connu pour être probablement le seul exemple d'un Bostonien ayant atteint le stade de l'immortalité à travers ce type d'écriture imagée. » (Scidmore 1885 : 274)

Le second totem - Chief Skowl's Totem Pole - est quant à lui mentionné pour la première fois en 1888 par Albert P. Niblack, qui le décrit ainsi :

«En face [de la maison de Skowl] se dresse sa colonne totémique, grande et finement sculptée, surmontée de l'emblème d'un aigle, l'emblème de Skowl. Au-dessous figure un homme, la main droite levée et l'index pointé vers le ciel. Son geste indique que Dieu - le dieu des Blancs - demeure dans les cieux. Plus bas encore se trouve la représentation d'un ange, tel que perçu par les Indiens d'après la description des Blancs, et ensuite apparaît un grand personnage censé représenter un missionnaire russe avec les mains pieusement croisées sur la poitrine. Cet ensemble [...] commémore l'échec des prêtres russes à convertir les gens de Skowl à leur foi, et fut érigé afin de ridiculiser et de rendre dérisoire la religion de l'homme blanc. » (1888, planche LV, fig. 294 : 378)

Cette interprétation de la signification du totem est corroborée soixante ans plus tard par l'anthropologue Viola Garfield : «Cette colonne sculptée est inhabituelle car un seul symbole traditionnel apparaît : l'aigle à son sommet. Les autres figures font écho à la défiance de Skowl à l'égard des efforts des Russes pour introedu/aipnw/wright.htm. 
Fig. 3 Mât totémique Abraham Lincoln, Saxman Totem Park, près de Ketchikan, Alaska @ $2010 \mathrm{Jim}$ Lavrakas/AlaskaStock.com.

\section{- $\bullet$}

3. On distingue généralement six catégories principales de totems, dotées chacune de fonctions particulières : les colonnes d'habitation ou mâts de façade, les mâts funéraires, les totems mémoriaux, les mâts héraldiques, les totems de potlatch et enfin les totems dits ridicules. duire leur religion dans le village de Kasaan. " (Garfield et Forrest 1948:67) Ces deux témoignages provenant de spécialistes reconnus semblent ne guère laisser de place à d'autres interprétations. Et pourtant, en 1963, paraît un ouvrage intitulé Monuments in Cedar. Son l'auteur, Edward L. Keithahn, propose une version en tout point différente de celle de ses deux prédécesseurs :

«Ce monument élaboré, d'inspiration pleinement européenne, fut érigé par le grand chef haïda, Skowl, à Kasaan au début des années 1880, et a depuis été déplacé à Ketchikan où il demeure visible dans le parc municipal. Il fut sculpté afin de commémorer le baptême du chef et de sa famille dans l'Église gréco-russe à New Archangel [Sitka]. Le style artistique inhabituel s'inspire des cartes représentants des saints, des chérubins, etc., données à Skowl par l'évêque russe. L'aigle surmontant le mât est le totem du chef Skowl; au-dessous se trouve la figure d'un saint russe; la troisième figure émergeant apparemment des nuages représente le visage de l'archange Michel, et au-dessous apparaît l'évêque russe. Ensuite vient un autre aigle, au-dessous duquel se trouve la figure du beau-fils de Skowl, Vincent Baranovich, un Autrichien de naissance [...]. La fille de Skowl, Mme Vincent Baranovich, finança en grande partie la sculpture, et il ne faisait aucun doute pour elle que la figure de son mari y était représentée. Il mourut en 1879 et Skowl durant l'hiver 1882-1883. " (1963: 143)

De symbole de la défiance des Indiens haïda à l'égard du prosélytisme des missionnaires russes, le totem, par un retournement de sens des plus surprenants, devient ainsi l'emblème de la conversion et de l'intégration de la famille Skowl au sein de l'église gréco-orthodoxe. Comme nous le vérifierons par la suite, une telle inversion de valeur n'est pas un phénomène unique. Ces deux exemples, empruntés à un corpus beaucoup plus large, permettent en outre de constater que la représentation totémique était généralement accordée à des figures et des personnalités non seulement américaines, mais aussi russes, rappelant par la même occasion l'étroite relation qui unissait auparavant marchands, religieux et colons de l'empire européen aux populations indigènes. Les interprétations contradictoires de ces figures européennes préfigurent les lectures antagonistes liées au totem Lincoln.

\section{La théorie du Grand Émancipateur selon James Wickersham}

Installés sur les côtes du Pacifique nord, dans les îles s'égrenant le long du sud de l'Alaska, ainsi que sur les rivages de la baie de Yakutat jusqu'au cap Fox, les Indiens tlingit ont produit un grand nombre de totems. Barbeau, qui consacra une partie de sa carrière à inventorier ces derniers, résume ainsi leur rôle et leurs fonctions au sein des sociétés qui les érigèrent : «Les figures sur les totems sont composées de symboles et d'illustrations, en grande partie comparables à notre héraldique, ou bien commémorant des événements historiques. Il ne s'agit pas de dieux païens ou de démons comme il est communément admis; ils ne sont jamais associés à un culte. D'habitude, ils illustrent des mythes ou des traditions tribales. Leur signification et leurs associations ont inspiré de la vénération plutôt que de la dévotion religieuse. " (1964:2) Arbres généalogiques ou monuments commémoratifs, les figures sculptées sur les totems identifient une lignée clanique et son histoire. La société tlingit est ainsi divisée en deux clans majeurs, celui des corbeaux et celui des aigles, et ces deux symboles jouent un rôle essentiel dans l'héraldique locale. Les autres animaux représentés sont quant à eux généralement associés à des génies protecteurs³ . 


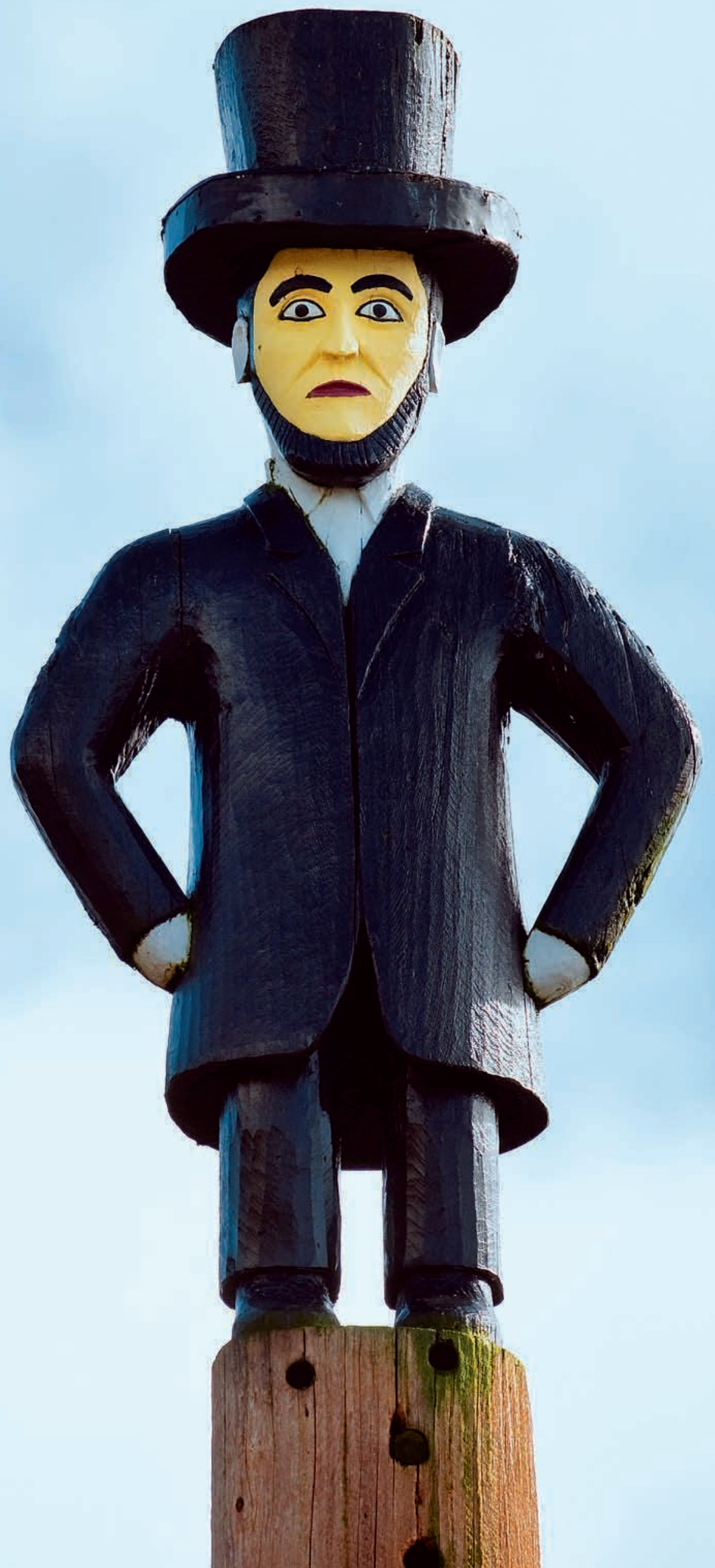




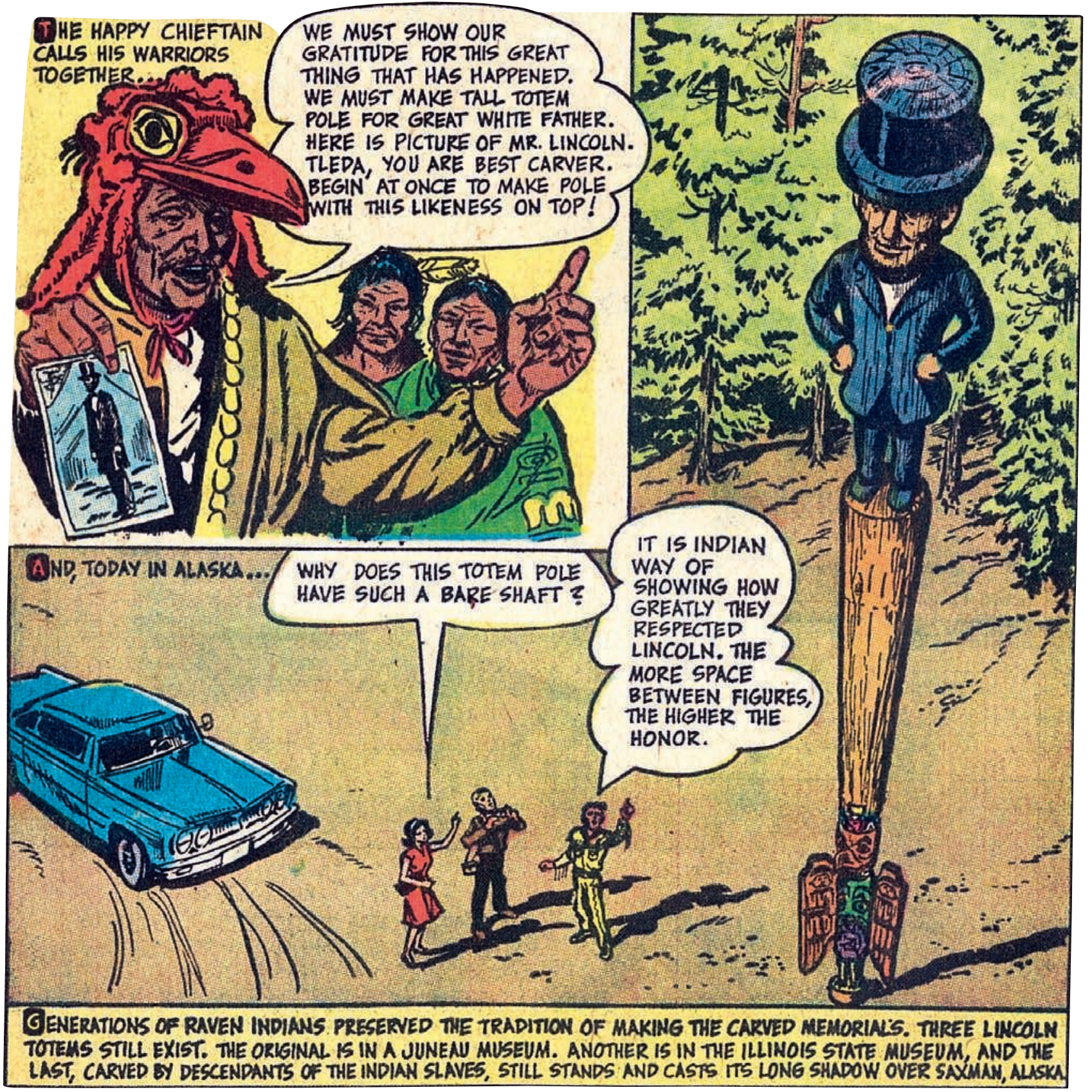

Fig. 4 Treasure Chest, vol. 23, n 4, p. 5, 19 octobre 1967. Avec l'aimable autorisation de la succession Lloyd Ostendorf, Dayton, OH, USA. 
En février 1924, le périodique Sunset Magazine fit paraître dans ses colonnes un court article signé Wickersham et intitulé "The oldest and rarest Lincoln statue ». Sunset Magazine était un journal mensuel consacré aux arts de vivre, au voyage et aux populations autochtones d'Amérique : cette étrange association, s'il en est, bénéficia pourtant d'un large lectorat, probablement attiré par quelques signatures prestigieuses dont celle de Jack London. Il ne se présentait pas comme une revue scientifique et privilégiait une approche divertissante destinée aux curieux et autres amateurs d'exotisme et d'aventures. L'auteur de cet article, James Wickersham (1857-1939), fut juge de district en Alaska avant d'être élu au Congrès américain en tant que représentant du même État. Sa contribution est, à notre connaissance, la première publication faisant mention du totem Lincoln : une page en tout et pour tout, illustrée de deux photographies montrant, sous un même angle et à quelques années d'intervalle, le totem in situ. Dès les premières lignes, l'auteur livre son interprétation :

«Ce mémorial est une statue totem du Grand Émancipateur réalisée il y a plus de cinquante ans [...]. L'histoire authentique de ce mémorial Lincoln sans équivalent prouve que la proclamation d'émancipation vint non seulement au secours des serfs noirs du Sud, mais dans son ombre, la tribu Tongass [i.e. les Indiens tlingit du village de Tongass] y trouva également asile et échappa ainsi à un terrible destin ayant pour finalité l'esclavage ou l'extermination. " (1924)

Wickersham décrit l'inimitié censée régner au sein des tribus tlingit entre le clan de l'aigle et celui du corbeau, auquel appartenaient les Indiens tongass. Plus nombreux et puissants, les membres du premier effectuaient régulièrement des raids sur le territoire des seconds, obligeant ces derniers à prendre la fuite, allant de refuge en refuge et ne trouvant jamais ni paix ni sécurité. Les Tongass auraient ainsi été réduits de moitié, victimes d'attaques meurtrières ou enlevés afin d'être réduits à l'état d'esclaves. Il est avéré qu'avant 1865, date de son abolition aux États-Unis, les Tlingit pratiquaient l'esclavage. La richesse d'un individu ou d'une famille se mesurait au nombre d'esclaves possédés. Ces derniers étaient capturés notamment chez les Aléoutes à l'ouest, ainsi que parmi les nombreuses tribus établies sur la côte pacifique jusqu'en Californie. Ils étaient échangés ou troqués comme n'importe quelle autre marchandise. Les témoignages sur le traitement qu'ils recevaient de leurs maitres varient cependant, insistant parfois sur une grande cruauté ou, au contraire, sur le caractère humain de certains propriétaires.

L'abolition de l'esclavage est contemporaine du rachat de l'Alaska par les États-Unis. Afin de gérer au mieux ce vaste territoire, le gouvernement américain installa un poste de douane ainsi qu'une compagnie militaire sur l'île de Kut-tuk-wah (aujourd'hui île Tongass), épaulé en mer par un navire de guerre - le Lincoln - dont l'une des missions consistait à faire cesser les hostilités entre Indiens. Apprenant cela, les Tongass, profitant d'un moment d'inattention de leurs ennemis, embarquèrent sur leurs canoës et s'installèrent sur la rive que protégeaient les canons du Lincoln. À la même époque (1869), William H. Seward, ancien gouverneur de New York et secrétaire d'État sous Lincoln, se rendit dans le village de Tongass. D'après Wickersham, il y fut chaleureusement accueilli et couvert de riches fourrures ${ }^{4}$. Les Tongass étaient en effet reconnaissants de leur protection aux officiers et aux militaires de l'armée et du service des douanes. Ils se sentaient particulièrement redevables envers l'équipage du Lincoln. Bien que la version de Wickersham soit relativement obscure à ce sujet, il semblerait que Seward leur aurait précisé que la présence salvatrice du navire avait été décidée
4. Pour célébrer la visite de Seward, un totem fut érigé à son image à quelques mètres du totem Lincoln. Plusieurs versions circulent également sur l'interprétation à donner à ce totem, dont une copie est aujourd'hui exposée au Saxman Totem Park. 
Fig. 5 Deux hommes tlingit, James Starrish et Charles Brown près de la statue sculptée de Lincoln, Saxman, Alaska. Collection Viola Garfield $n^{\circ} .130$, Washington University Libraries. Special Collections Division. par Lincoln lui-même, «le grand chef de la nation sous le drapeau de laquelle ils vivaient désormais, enfin libérés du joug de l'esclavage " (Wickersham 1924). En guise de remerciement, Ebbets, l'un des anciens chefs du village, aida Tsa-kad, son successeur, à faire ériger un nouveau totem en l'honneur du grand homme de Washington. Sa réalisation en fut confiée au sculpteur Old Thle-da, à qui l'on donna une photographie ou une image de Lincoln pour modèle. Wickersham termine son article par cette émouvante requête :

«Lors d'une récente visite en Alaska, le Président Harding avait reçu une demande visant à faire de l'île de Tongass un monument national afin de préserver ce mémorial unique et sans prix ainsi que le groupe de totems qui s'y trouve. Encore quelques années, et les totems tomberont ou seront volés [...], et alors la statue de Lincoln la plus vieille et la plus rare sera perdue. Le peuple des États-Unis devrait donc demander au Président Coolidge [qui avait succédé en 1923 à Harding, décédé brusquement après deux années de mandat] de la sauver pendant qu'il en est encore temps. » (Ibid.)

Ainsi, si l'on en croit Wickersham, les Tlingit du village de Tongass auraient érigé ce totem afin de rendre hommage au président américain qui avait mis un terme à une longue période d'insécurité et d'esclavage. L'homonymie entre le navire de guerre pacificateur et le nom du président facilita certainement les choses, tout en contribuant à brouiller les pistes. En quelques années, cette interprétation fit florès puisque successivement, en 1947 et 1948, parurent deux articles qui s'inspiraient de la version de Wickersham. Le premier fut publié dans le numéro de février 1947 de la revue Natural History. Il était signé Virginia S. Eifert, éditrice à l'Illinois State Museum et auteur de plusieurs ouvrages sur l'histoire et la carrière de Lincoln. L'article reprenait en les développant les informations de Wickersham, tout en y ajoutant quantité de détails parfois fantaisistes. L'auteur évoquait notamment, sans la moindre preuve, le fait que plusieurs tribus voisines du village de Tongass, libérées du joug de l'esclavage, auraient également fabriqué des totems en l'honneur de Lincoln. Cela est d'autant plus improbable qu'aucune trace de ces mâts n'a été retrouvée à ce jour. L'enthousiasme prosélyte d'Eifert à l'égard du président américain ne paraît finalement que faire écho au temps et à l'énergie qu'elle consacra toute sa vie à la mémoire du grand homme.

Eifert livre cependant une information intéressante, mais en partie inexacte : «Trois exemplaires des vieux totems Lincoln sont encore connus à ce jour. Un au musée de Juneau, un autre dans le petit village indigène de Saxman en Alaska, et un troisième à l'Illinois State Museum. » (1947 : 66) En réalité, seul le premier de ces totems fut érigé à Tongass. Les deux autres sont des copies, dont l'une trône aujourd'hui encore à l'entrée du musée éponyme de Springfield, dans la capitale de l'Illinois, tandis que le second, régulièrement reproduit en carte postale, est visible au Saxman Totem Park. La copie de Springfield, ville où Lincoln débuta sa carrière d'avocat en 1837 et où sa dépouille est aujourd'hui enterrée, fut acquise en Alaska par des représentants du musée. Elle s'ajoute aux nombreux monuments à la gloire du président américain construits dans cette ville et en font un lieu de pèlerinage pour les admirateurs du grand homme.

En 1948, l'anthropologue Viola E. Garfield et l'architecte Linn A. Forrest publient The Wolf and the Raven: Totem Poles of Southeastern Alaska, dans lequel apparaissent trois reproductions du totem Lincoln. Elles précisent :

«Un artiste tsimshian de Port Simpson, en Colombie-Britannique, du nom de Thleda, fut engagé pour réaliser la sculpture. À la base se trouve une excellente figure de corbeau, appelée Proud Raven ["Corbeau fier»], honorant le clan des corbeaux de 


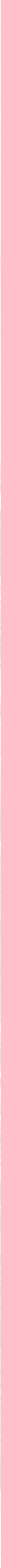


-

5. «Le totem historique ou commémoratif, comme son nom l'indique, raconte des événements particuliers et importants (ou en tout cas considérés comme tel par le propriétaire du mât] dans I'histoire de la famille ou du chef de maison. En général, il s'agit de récits de conflits entre l'homme et l'animal, et les dénouements victorieux sont chroniqués sur ces monuments pour l'édification des générations futures. » (Jones 1970 : 176-177) Ce type de totem peut également faire référence à l'arrivée de l'homme blanc sur le territoire indien.
Tongass qui fut plus malin que celui des Kagwantan [le clan de l'Aigle] et le força à faire la paix. Le totem est généralement connu chez les Tlingit sous le nom de Proud Raven Pole. On donna à Thleda une image de Lincoln qui lui servit de modèle pour le haut de la colonne. » $(1948: 55)$

Cette dernière version est moins catégorique quant au rôle d'émancipateur de Lincoln, même si elle ne remet pas véritablement en cause la thèse de Wickersham. L'approche de Garfield et Forrest se garde de toute tentative de surinterprétation: «Le totem commémorait la fin des hostilités entre deux villages tlingit et symbolisait l'espoir de paix et de prospérité qui devait suivre l'occupation du territoire par les Américains. " (Ibid. : 54) L'ouvrage fait également mention d'un bâtiment de la marine américaine (le Lincoln), qui aurait assuré la sécurité des Indiens du village de Tongass, ainsi que d'une photographie du président qui aurait servi de modèle au sculpteur. La version apologétique du mât proposée par Wickersham semble ici ramenée à de plus justes proportions. Elle annonce une série d'articles et de prises de position qui s'écartent plus encore de l'interprétation initiale.

\section{William Paul et le premier homme blanc}

Natif du village de Tongass, William Paul (1885-1977), avocat, activiste politique et leader de l'Alaska Native Brotherhood, ne cessa de s'opposer à la version de Wickersham. Dans un article publié en 1971 par The Alaska Journal, et qui demeure à ce jour le texte le plus complet sur le totem Lincoln, il évoque avec force détails les arguments qu'il oppose à la théorie du Grand Émancipateur. D'après ses sources, le totem fut érigé dans le village de Tongass en 1883. Au cours des années qui suivirent l'érection du mât, de nombreux voyageurs, parmi lesquels plusieurs écrivains et anthropologues, eurent l'occasion de s'arrêter dans le village, mais aucun ne releva la présence du totem ou quelque histoire se rapportant au président américain. Ce constat s'explique, selon Paul, par un argument imparable : "Je suis certain que la raison pour laquelle ils ne mentionnent pas cette histoire, c'est que celle-ci n'avait pas encore été inventée. " (1971: 6)

Paul avait reçu en 1922 un courrier de Wickersham sollicitant des informations à propos du fameux totem : le futur élu au Congrès «ne cherchait pas à savoir si son histoire était juste, mais uniquement des preuves pour la confirmer " (ibid.). Fort affairé à l'époque, Paul ne prit pas le temps d'y répondre et c'est ainsi que l'histoire de Wickersham devint la version la plus répandue. Certainement blessé de voir un individu n'appartenant pas à la communauté s'accaparer l'histoire des Tlingit au profit d'une glorification de Lincoln, Paul décida à son tour de mener l'enquête. Il retrouva d'anciens habitants du village de Tongass, mais aucun ne connaissait d'histoires à propos d'un totem lié à l'esclavage. Néanmoins, «une fois que nous parvînmes à identifier le totem en question, connu d'eux sous le nom de Proud Raven Pole, ils m'en racontèrent l'histoire " (ibid. : 8). Le mât n'était pas entièrement sculpté, mais possédait à sa base la figure d'un corbeau et à son sommet la silhouette d'un homme. Il s'agissait manifestement d'un totem commémoratif5. Au cours du xix siècle, les Tlingit avaient en effet pris l'habitude d'ériger des totems pour marquer des événements importants de leur histoire. Le village de Tongass abritait ainsi un totem surmonté de la figure d'un homme blanc assis sur une caisse, qui commémorait la visite de Seward en 1869. Un autre totem affichait un navire occidental, référence à la première fois où un voilier fut aperçu le long des côtes. 


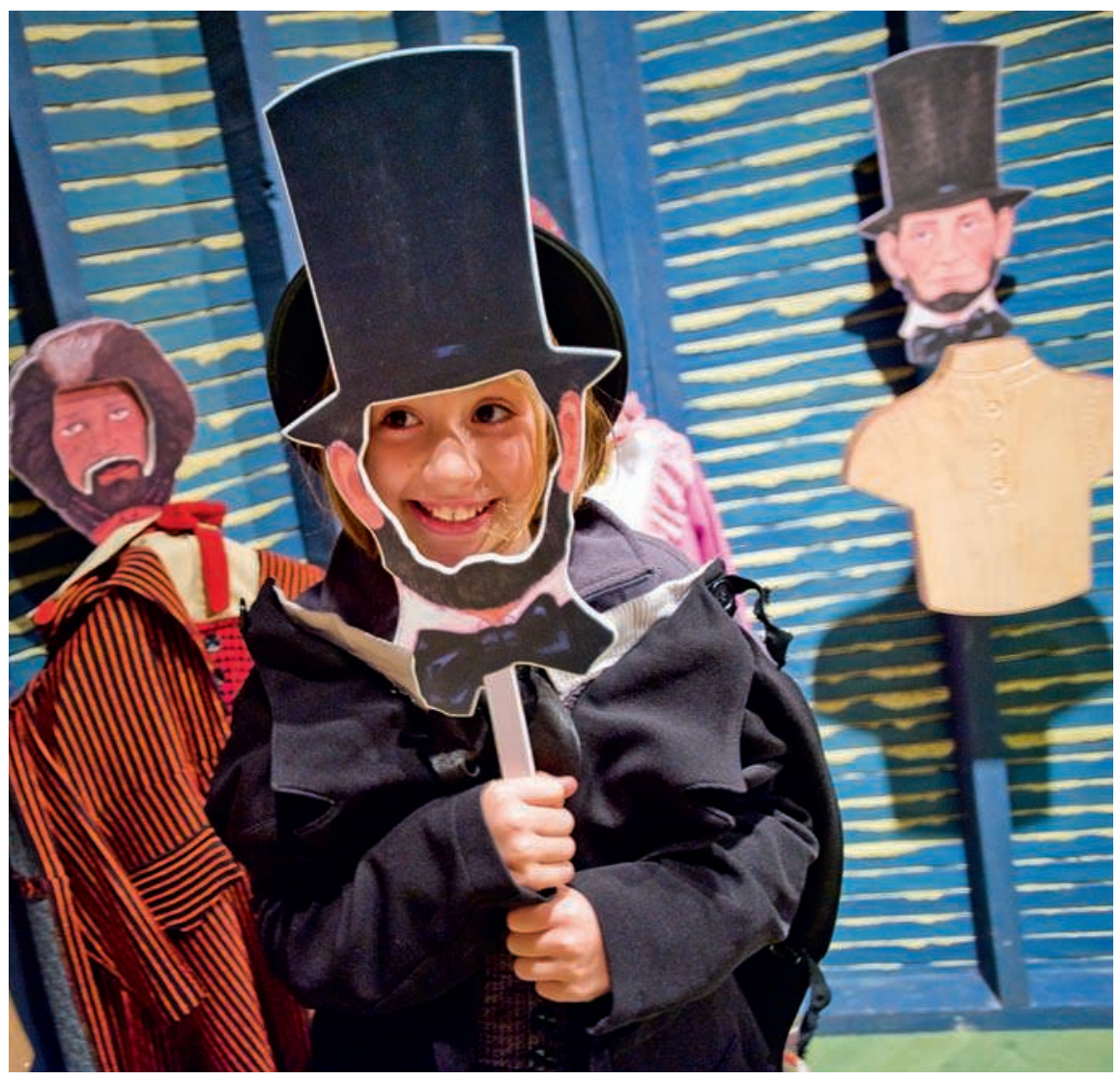

Fig. 6 Atelier pour enfants au musée Lincoln. Avec l'aimable autorisation du Abraham Lincoln Presidential Library and Museum.

Il en allait de même du Proud Raven Totem : il avait été commandé pour un certain Yahl-jeeyi, un chef appartenant au clan du corbeau, qui était très fier que lui ou l'un de ses ancêtres (la version recueillie par Paul n'est pas claire sur ce point) fût le premier homme de la région à voir un Occidental, probablement un explorateur ou un marchand. Le chef fit appel à un sculpteur tsimshian afin de réaliser un totem commémorant cet événement, avec l'emblème de son clan - un corbeau - à la base du mât et un homme blanc à son sommet. Le sculpteur ayant besoin d'un modèle pour l'homme blanc, un habitant du village lui transmit une photographie qui lui avait été donnée, d'après la tradition, par l'officier commandant le poste de l'armée installé à Tongass de 1868 à 1870 et sur laquelle était représenté le président américain. En quelque vingt pages, Paul reprend point par point les faits narrés par Wickersham pour démontrer leur inexactitude. Il conclut ainsi son article :

"Aujourd'hui la figure originale du totem de l'île Tongass repose à l'Alaska State Museum à Juneau, tandis qu'une réplique du mât, sculptée il y a trente ans par des artisans du Civilian Conservation Corps, se trouve au parc à totems de Saxman, près de Ketchikan. Appelez-le comme vous le désirez - Totem Lincoln, Proud Raven Totem, First White Man Totem -, ces noms rappellent qu'un événement historique important se déroula sur ces rivages. Mais seule une infime partie de l'histoire est connue [...], la plus grande partie demeurant à jamais perdue dans la nuit des temps. " (Ibid. : 16) 
Fig. 7 Des étudiants jouent avec une tête d'Abraham Lincoln dans la rotonde du Capitole. Washington D.C., 1957 (C) Henri Cartier-Bresson/ Magnum Photos.
En 1976, le Journal of American Folklore publia en couverture de son volume LXXXIX deux photographies de la figure de Lincoln conservée au musée de Juneau. La légende qui accompagnait l'iconographie faisait uniquement référence à la version de Paul, tout en précisant qu'il s'agissait d'une source indigène. Le monde scientifique, sans prendre formellement position, semblait ainsi accréditer la version de Paul au détriment de celle de Wickersham.

\section{La figure de la honte}

Il faudra attendre encore vingt-six ans pour qu'apparaisse une troisième version, proposant une lecture inédite et pour le moins surprenante du totem Lincoln. Le 5 juillet 1997, l'Anchorage Daily News publiait sous la plume du journaliste et écrivain Tom Kizzia un article intitulé "One totem, many stories». Au cours d'une conférence devant les membres de l'Alaska Federation of Natives (AFN), la plus importante association autochtone d'Alask $\mathrm{a}^{6}$, au sujet d'un différend sur la souveraineté tribale, le sénateur Robin Taylor cita le président Lincoln comme symbole de la lutte pour l'intégration et l'assimilation. Évoquant le fameux totem, il reprit la version de Wickersham, mais en l'attribuant à Paul. L'affront, bien qu'il ne fût sans doute pas volontaire, était de taille puisque Paul avait participé à la fondation de l'AFN. Une anthropologue tlingit, Rosita Worl, à la tête de la Sealaska Heritage Foundation, intervint pour préciser que, d'après la tradition orale, le totem Lincoln avait été sculpté par des chefs furieux d'avoir perdu leurs esclaves sans la moindre compensation : «Les esclaves étaient une forme de propriété, et ce bien équivalait à une prime. Quand les esclaves furent affranchis, une dette fut ainsi créée. " (Kizzia 1997) Le totem Lincoln appartiendrait donc à la catégorie des totems dits "ridicules " (ridicule ou discredit poles). Lorsque certains chefs tlingit voulaient manifester leur colère ou leur réprobation, ils faisaient parfois ériger un totem commémorant, par exemple, une dette impayée. C'était une manière habile, et particulièrement visible, de faire honte à son ennemi ou à son débiteur. Kizzia rappelle que cette tradition a toujours cours le long des routes américaines, où les propriétaires de stations-services n'hésitent pas à afficher sur des enseignes le nom des clients indélicats ayant signé des chèques en bois.

Barbeau recense trois exemples de totems dits ridicules chez les Tlingit, dont le plus important semble être un mât érigé sur Chief Shaiks' Island à Wrangell :

"Le meilleur exemple d'un totem ridicule encore existant est celui reconstruit à Wrangell [...]. Il se compose d'un perchoir en forme de T sur lequel sont juchées trois énormes grenouilles. Elles représenteraient trois femmes kiksadi (dont le totem est la grenouille) qui auraient vécu avec des esclaves appartenant au chef nommé Shakes [Shaiks]. Après un certain temps, ce dernier se rendit auprès du chef des Kiksadi en exigeant une rétribution pour l'entretien de ces trois femmes qui vivaient dans sa domesticité. Le chef kiksadi refusa cependant de payer, arguant que les femmes s'étaient abaissées elles-mêmes en contractant une union au-dessous de leur condition et avaient donc été chassées de la tribu. Shakes ne fut pas satisfait d'une telle réponse et, d'après la tradition, il fit sculpter un totem ridicule afin de forcer le paiement. L'histoire ne dit pas si la dette fut honorée ou non. » (1964 : 399-401)

Il existe par ailleurs dans le village de Kake un totem dit ridicule représentant un assassin russe. À Sitka, enfin, un totem du même type présenterait un Occidental sous la forme d'un corbeau, totem censé faire honte à un commerçant blanc qui, après avoir été adopté par le clan du Corbeau, aurait refusé (ou n'aurait pas été en mesure) de répondre au potlatch fait en son honneur par les Indiens tlingit. 


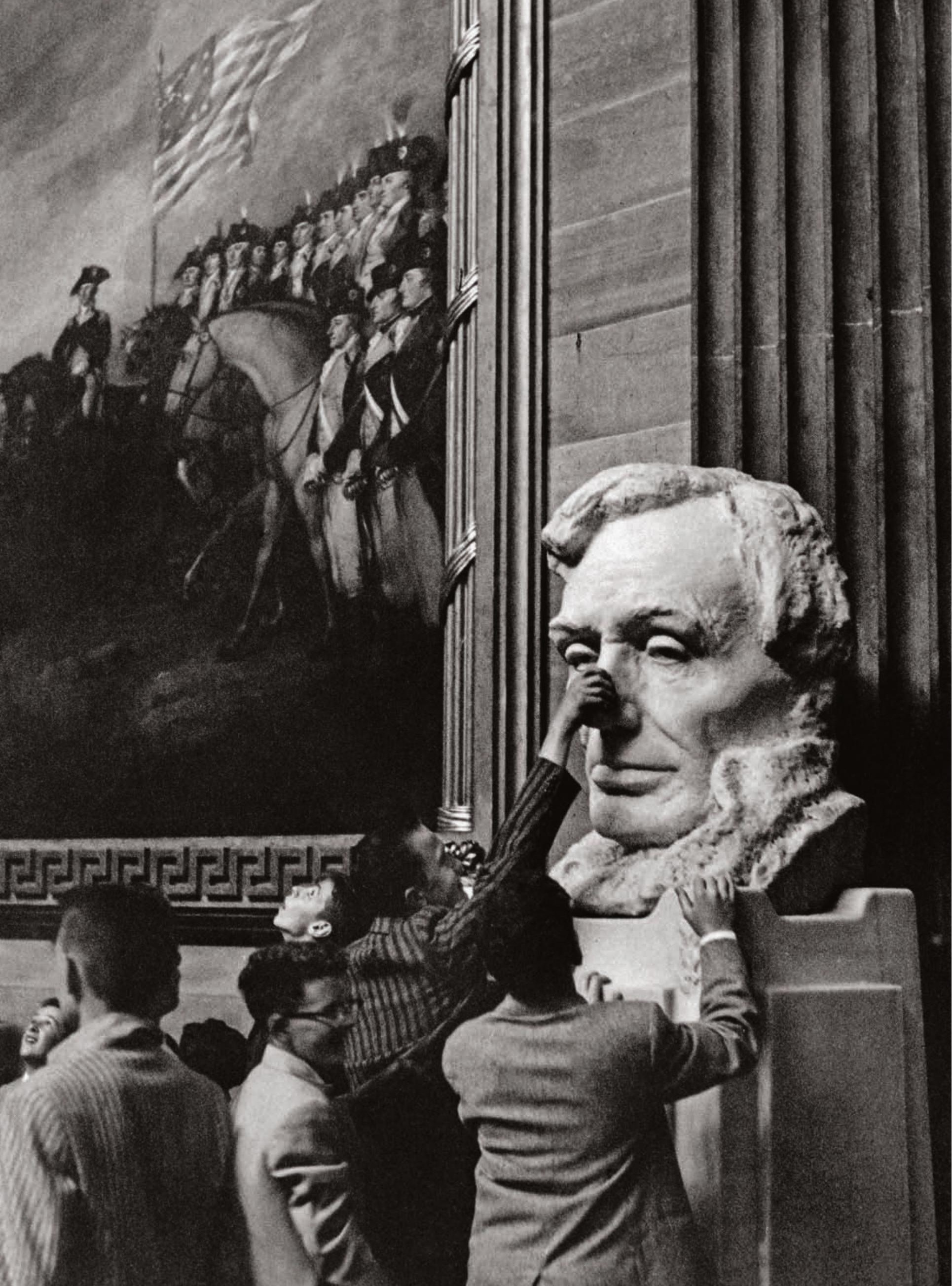


Réinterprétant le totem Lincoln à l'aune de cette catégorie des totems dits ridicules, Worl conclut ainsi son intervention au cours de la conférence du sénateur Taylor : "Vous entendez peu parler de cette histoire car les Tlingit ne sont pas fiers d'avoir possédé des esclaves.» (Kizzia 1997)

À propos d'un même totem, trois versions se sont donc succédé, trois lectures contradictoires comme autant de perspectives ouvertes sur les relations entre le gouvernement américain et les Indiens tlingit d'Alaska. Dans un premier temps, la figure de Lincoln est associée à celle du Grand Émancipateur, figure paternelle et protectrice venue pacifier et unifier une région en proie à des querelles intertribales. Cette thèse émise par un homme politique blanc vient légitimer la présence américaine sur le sol indien. Ni Paul ni Worl n'accordent la moindre foi à cette version. Leur appartenance revendiquée au peuple tlingit fait de leur opposition un geste non seulement culturel, mais également politique. Paul, qui consacra indubitablement le plus d'énergie à l'étude du mât et qui était le plus acharné à décrédibiliser la thèse de Wickersham, se garde pourtant de tout jugement péremptoire. Il préfère désacraliser le totem pour n'en conserver symboliquement que la trace de l'apparition du premier homme blanc, événement certes considérable dans l'histoire des Indiens de Tongass, mais déchargé par la même occasion de toute fonction apologétique à l'égard de Lincoln et donc du pouvoir politique américain dans la région. L'avocat fut parmi les premiers défenseurs des droits autochtones et sa position, finalement modérée, n'apparaît que comme l'amorce de revendications beaucoup plus radicales. La version de Worl fait du totem une trace de la dette de l'homme blanc envers les Indiens, image d'autant plus paradoxale qu'elle fait passer Lincoln de la figure de Grand Émancipateur à celle de Grand Débiteur des habitants de Tongass. Étrange cheminement où une sculpture, en fonction des époques et des regards, semble signifier une chose et son contraire. L'image du pouvoir fédéral américain y apparaît sous deux jours antagonistes, tantôt source de bienfait, tantôt source de préjudice, ouvrant ainsi la voie à une possible relecture systématique de l'art des peuples indigènes d'Alaska à travers le prisme de la présence de l'homme blanc.

musée du quai Branly nme@quaibranly.fr

mots clés / keywords : totem // totem • Tlingit // Tlingit • Lincoln // Lincoln • esclavage // slavery • dette // debt • Alaska // Alaska $\cdot$ premiers contacts // first contact. 


\section{Bibliographie}

\section{BARBEAU, Marius}

1964 Totem Poles. Ottawa, National Museum of Canada.

\section{Bolanz, Maria et Williams, Gloria C.} 2003 Tlingit Art. Totem Poles and Art of the Alaskan Indians. Blaine, Hancock House Publishers.

\section{EIFERT, Virginia Louise Snider}

194 « Lincoln on a Totem Pole. The strange story of how the Tlingit Indians honored the man who freed the slave », Natural History (56)2 : 64-66.

\section{EmMons, Georges T.}

1914 «Portraiture among the North Pacific Coast tribes », American Anthropologist, janvier-mars.
Garfield, Viola et Forrest, Linn A. 1948 The Wolf and the Raven: Totem Poles of Southeastern Alaska. Seattle, University of Washington Press.

\section{JoNes, Livingston F.}

1970 A Study of the Thlingets of Alaska. New York, Fleming H. Revell.

1976 Journal of American Folklore (89)353, juillet-septembre.

\section{KeITHAHN, Edward L.}

1963 Monuments in Cedar. The authentic story of the Totem Pole. New York, Bonanza Books.

\section{KizZIA, Tom}

1997 « One totem, many stories », Anchorage Daily News, 5 juillet.

\author{
LiPs, Julius \\ 1937 The savage hits back. New Haven, \\ Yale University Press. \\ Niblack, Albert P. \\ 1888 The Coast Indians of Southern Alaska and \\ Northern British Columbia. Report of the U.S. \\ National Museum. \\ PAUL, William \\ 1971 «The Real Story of the Lincoln Totem », \\ The Alaska Journal, été : 2-16. \\ SCIDMORE, Eliza Ruhamah \\ 1885 Alaska. Its Southern Coast and the Sitkan \\ Archipelago. Boston, D. Lothrop Company.

\section{WicKeRSHAM, James} \\ 1924 «The oldest and rarest Lincoln statue », \\ Sunset Magazine (52)2, février : 35.
}

\section{Résumé / Abstract}

Nicolas Menut, Les vérités contrariées du totem Lincoln - Au cours des années 1870 dans le village de Tongass, en Alaska, est érigé un totem tlingit au sommet duquel trône la figure du président américain Abraham Lincoln. Depuis lors, anthropologues, administrateurs gouvernementaux et Tlingit ont avancé des interprétations divergentes afin d'expliquer cette présence incongrue au faîte d'un totem indien. Tour à tour associé à la figure du Grand Émancipateur, puis du premier homme blanc avant d'être finalement ramené au rôle de Grand Débiteur, le mât Lincoln est indissociable de la réappropriation par les Indiens tlingit du sens de leur propre histoire et des contacts complexes qu'ils entretinrent avec les hommes blancs.
Nicolas Menut, The Vexed Truths of the Lincoln Totem - During the 1870s, a Tlingit totem was erected in the village of Tongass in Alaska. At its crown was the likeness of the American president, Abraham Lincoln. Since then, anthropologists, government administrators and Tlingit themselves have put forward varying interpretations of his incongruous presence at the summit of an Indian totem pole. Initially understood as symbolising the great liberator, he was later interpreted as the first white man, before finally being seen as the great debtor. This progression is closely linked to Tlingit Indians reappropriation of the meaning of their own history and of the complex nature of their relationships with white men. 

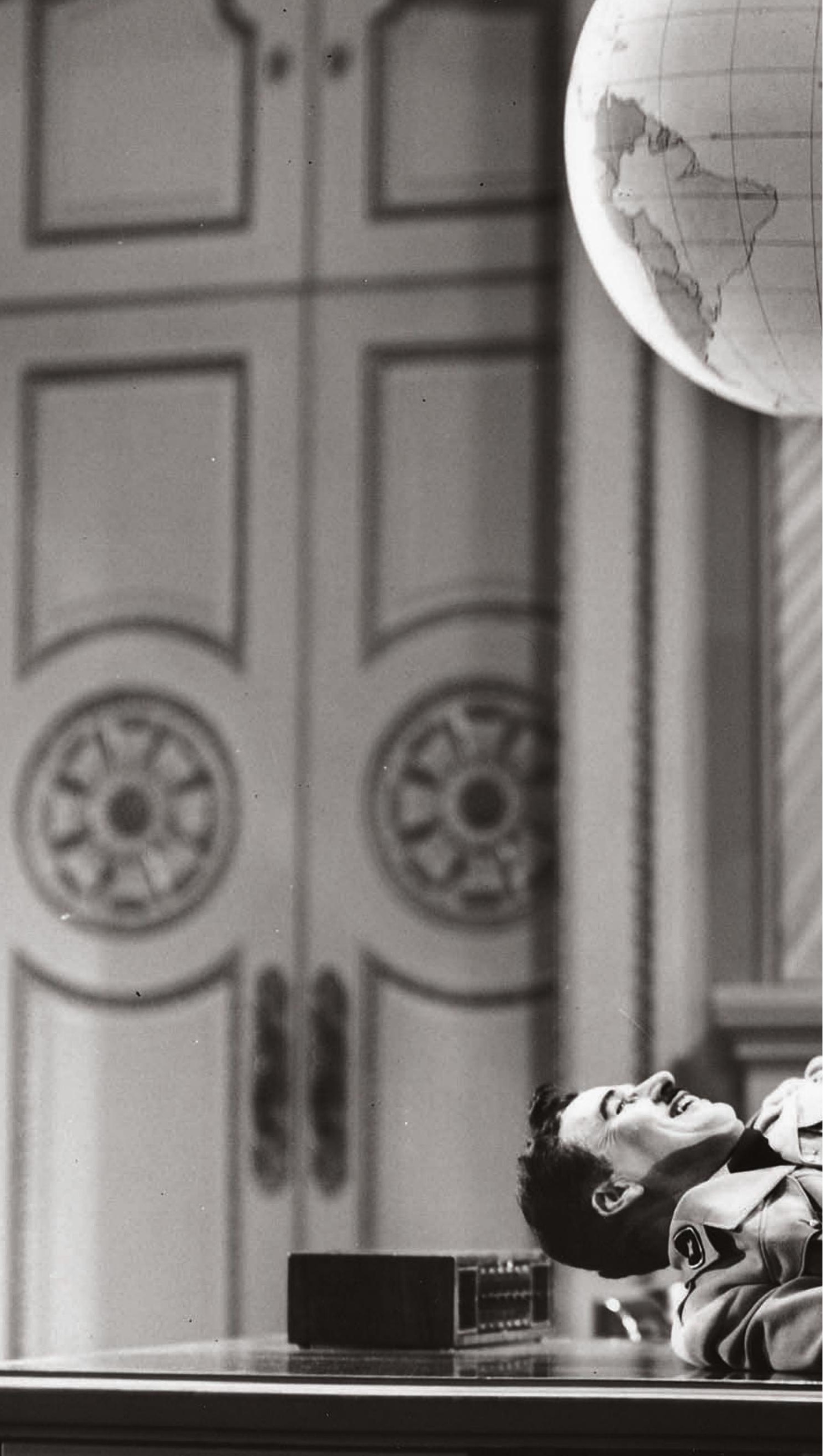
